\title{
A Three-Class Procedure for Acceptance Sampling by Variables
}

\author{
P. A. Brown and 0. B. Allen
}

$\mathrm{BU}-891-\mathrm{M}$

September 1985

\section{Abstract}

A three-class procedure for acceptance sampling by variables is introduced as an alternative to both the three-class attributes plans introduced by Bray et al. (1973) and to the two-class variables plans introduced by Jennett and Welch (1939). The procedure, which requires that the quality characteristic be normally distributed, has the advantage of requiring a smaller sample size than a three-class attributes plan with approximately the same OC surface. The advantage of the three-class variables plan over the two-class variables plan is a greater ability to discriminate between conforming and non-conforming lots. Two equivalent methods of stating the decision rules for the plans are suggested. It is shown that the operating characteristic surface for the three-class variables procedure may be constructed with a special case of the bivariate noncentral tdistribution. Motivation and methods for choosing a plan are discussed and illustrated.

KEY WORDS: Acceptance sampling; Attributes plans; Variables plans; Three-class plans; Bivariate noncentral t-distribution. 


\section{INTRODUCTION}

Consider the setting in which a lot of items are to be either accepted or rejected based on a quality characteristic which can be measured on each item in the lot. We consider the case in which the quality characteristic is a quantitative measurement. (We assume throughout that quality is a decreasing function of this measurement.) Typically, a sample of items is drawn from the lot, the quantitative measurement made on each item sampled and a decision made to reject or accept the lot based on these measurements. In order to use two-class attributes plans (Koyoma, 1970) each sampled item must first be categorized as conforming or non-conforming. This requires that a limit be established for the quantitative measurement, below which items are conforming and above which items are non-conforming.

An attributes plan bases the decision to accept or reject the lot only on the number of items exceeding this limit. A plan can then be chosen to guarantee that a lot will be rejected with high probability if a sufficiently high proportion of items in the lot exceed the chosen limit. Variables plans, introduced by Jennett and Welch (1939) and reviewed by Kao (1971), are able to achieve the same control with a smaller sample size by making use of the distribution of the intial measurements.

In many acceptance sampling settings, the distinction between a conforming and non-conforming item is not sharp. This is the case with the microbiological testing of foods, for example. Indicator organisms are microorganisms which do not themselves pose a direct health hazard but whose presence is easy to identify and indicates that conditions may have existed which would promote the grow of pathogenic microorganisms. A high count, for example, might indicate that the food had undergone poor 
handling or storage conditions. For such organisms a low count may be acceptable but a sufficiently high count may be grounds for rejection. However, there is no sharp demarcation between what is acceptable and what is not. There may be a range of values between conforming and nonconforming for which the item is considered marginally conforming. Bray et al. (1973), recognizing this problem, introduced three-class attributes plans. These plans have two limits which divide bacterial counts into three classes - conforming, non-conforming and marginally conforming. The quality of the lot is determined by both the proportion of non-conforming and the proportion of marginally conforming items it contains. A two-class acceptance sampling procedure does not have the ability to discriminate adequately amongst different lot qualities when defined in this way. These three-class attributes plans have been well received in the food industry (ICMSF, 1978).

In the present paper, a three-class procedure for acceptance sampling by variables is introduced. It is analogous to the three-class attributes plan with respect to its definttions of item and lot quality. Two plans may be considered equivalent if their respective operating characteristic surfaces are identical. In general, although it is not possible to construct a variables plan for which the surface is coincidental with the surface of a specified attributes plan at all points, it is possible to match the two plans so that their surfaces touch at certain suitably chosen points. This is the approach taken here to develop the three-class variables procedure as an alternative to the three-class attributes procedure. The procedure for variables has the usual advantage of requiring a smaller sample size than an equivalent procedure for attributes but it does require that the quality characteristic have a normal distri- 
bution (or a distribution transformable to a normal distribution). It must be remembered, though, that the cost of obtaining the precise measurements required for the variables procedure may be higher than the cost of obtaining the observations required for the attributes procedure. The advantage of the variables procedure is real only if the entire cost of the sample (and not just the sample size) is reduced.

The nature of the quality characteristic being measured must be such that two upper specification limits, $U_{1}$ and $U_{2}$ (with $U_{1}<U_{2}$ ), may be stated. An item will be considered to be marginally conforming if its measurement is between $U_{1}$ and $U_{2}$ and to be non-conforming if its measurement is above $U_{2}$. The cholces of $U_{1}$ and $U_{2}$ are technical problems which must be dealt with by individuals with expertise in the particular manufacturing process under consideration. The lot quality will be defined in terms of $p_{1}$ and $p_{2}$ where $p_{1}$ is the proportion of 1tems in the lot with measurements exceeding $U_{1}$ and $p_{2}$ is the proportion with measurements exceeding $\mathrm{U}_{2}$. The theory for two lower specification limits will be simflar due to symmetry; the two-sided case, which would involve four specification limits (lower and upper marginal limits as well as lower and upper non-conforming limits) is not considered here.

It is natural to consider a three-class plan in the case in which the quality characteristic follows a normal distribution (or is transformable to a normal distribution). Since the normal distribution has two parameters, by specifying $\left(U_{1}, U_{2}\right)$ and $\left(p_{1}, p_{2}\right)$ one specifies a unique underlytng distribution. In the case of the two-class plans, there are an infinite number of distributions for which a proportion $p$ exceeds $U$. Some of these distributions may be regarded as more undesirable than others. 
The acceptance criterion for the proposed plans may be stated in one of two ways, which will be referred to as the $\left(k_{1}, k_{2}\right)$-method and the $\left(p_{1}^{*}, p_{2}^{*}\right)-$ method. These two methods are equivalent for the same sample size, and for either one, the operating characteristic surface is constructed using a special case of the bivariate noncentral t-distribution.

\section{THREE-CLASS VARIABLES PLANS}

A sample of $n$ items is selected at random from a lot and the mean, $\bar{x}$, and standard deviation, s, are computed. The decision rule is to accept the lot only if both $\bar{x}+k_{1} s \leq U_{1}$ and $\bar{x}+k_{2} s \leq U_{2}$ and to reject the lot otherwise, for suitably chosen values of $k_{1}$ and $k_{2}$ (with $\left.k_{1}<k_{2}\right)$. The probability of acceptance of a lot of arbitrary quality $\left(p_{1}, p_{2}\right)$ is thus

$$
\begin{aligned}
& P\left(\bar{x}+k_{1} s \leq U_{1} \text { and } \bar{x}+k_{2} s \leq U_{2} \mid p_{1}, p_{2}\right) \\
& =P\left(T_{1 f} \leq-k_{1} n^{\frac{1}{2}} \text { and } T_{2 f} \leq-k_{2} n^{\frac{1}{2}} \mid \delta_{1}, \delta_{2}\right)
\end{aligned}
$$

where $T_{1 f}=\sqrt{n}\left(\bar{x}-U_{1}\right) / s$ has a noncentral t-distribution with $f=n-1$ degrees of freedom and noncentrality parameter $\delta_{i}=-n^{\frac{1}{2}} k_{p i}$, and $k_{p i}$ is the $100\left(1-p_{1}\right)$ th percentile of the standard normal distribution, $1=1,2$. The joint distribution of $T_{1 f}$ and $T_{2 f}$ is a special case of the bivariate noncentral t-distribution. Owen (1965) used this distribution for applications to two-sided tolerance limits and to two-sided variables sampling plans. Details necessary to evaluate (1) are given in the appendix.

A point on the OC surface will be identified by a vector of the form $\left[p_{1}, p_{2}, p_{a}\left(p_{1}, p_{2}\right)\right]$, where $p_{1}$ and $p_{2}$ are as defined earlier and $p_{a}\left(p_{1}, p_{2}\right)$ is the probability of acceptance of a lot of this quality. The approach for choosing $n, k_{1}$ and $k_{2}$ is to specify three points on the OC surface. This 
uniquely determines $n, k_{1}$ and $k_{2}$. The procedure is summarized in the following three steps:

Step 1: Specify the following three points on the OC surface:

(i) When $p_{1} \leq a$ and $p_{2}=0$, the probability of acceptance is at least $1-\alpha$.

(1i) When $p_{1} \geq b$ and $p_{2}=0$, the probability of acceptance should be at most $\beta$ where $b>a$ and $\beta\langle 1-\alpha$.

(iii) Finally when $\mathrm{p}_{1} \geq \mathrm{c}$ and $\mathrm{p}_{2} \geq \mathrm{c}$, the probability of acceptance should be at most $\gamma$ where $\gamma<P\left(\bar{x}+k_{1} s \leq U_{1} \mid p_{1}=c\right)$. Since the values of $n$ and $k_{1}$ are determined by (1) and (ii) (see Step 2), this probability is completely determined by $a, b, \alpha$ and $\beta$. For example, if $c=a$, then $\gamma<1-\alpha$ and if $c=b$ then $\gamma<\beta$. Thus, the three points on the oC surface which have been specified are $(a, 0,1-\alpha),(b, 0, \beta)$ and $(c, c, \gamma)$.

Step 2: Find $n$ and $k_{1}$ so that points (i) and (ii) above are satisfied as closely as possible. When $p_{2}=0$ the probability of acceptance is independent of $k_{2}$ and the problem reduces to finding $n$ and $k_{1}$ for $a$ two-class plan for which the oc curve passes through the two points $(a, 1-\alpha)$ and $(b, \beta)$. Using Hamaker's (1979) approximate method, $n$ and $k_{1}$ are the solution to

$$
\begin{aligned}
& n=n_{\sigma}\left(1+k^{2} / 2\right)+1 \\
& k=k_{\sigma}(4 n-4) /(4 n-5)
\end{aligned}
$$

where the appropriate values of $n$ and $k$ if $\sigma$ were known are (Wallis, 1947)

$$
\begin{aligned}
& n_{\sigma}=\left[\left(k_{\alpha}-K_{1-\beta}\right) /\left(K_{a}-K_{b}\right)\right]^{2} \\
& k_{\sigma}=\left(K_{a} K_{1-\beta}-K_{b} K_{\alpha}\right) /\left(K_{1-\beta}-K_{\alpha}\right),
\end{aligned}
$$

where $\Phi\left(\mathrm{K}_{\mathrm{x}}\right)=1-\mathrm{x}$ and $\Phi(\cdot)$ is the cdf of the normal $(0,1)$ distribution. More precise values for $n$ and $k$ can be determined tteratively from the noncentral t-distribution using a procedure by Wallis (1947, pp. 81-82). 
Step 3: Using the sample size determined in step 2, find a value of $\mathrm{k}_{2}$ so that point (iii) above is also satisfied. When $p_{1}=p_{2}$, the probability of acceptance is independent of $k_{1}$ and the problem again reduces to a two-class situation. The value of $k_{2}$ is chosen so that for the sample size, $n$, determined in step 2 , the point $(c, \gamma)$ is on the resulting oC curve. The exact value of $k_{2}$ is equal to $-t_{0} / n^{\frac{1}{2}}$ where $t_{0}$ is the $100 \gamma$ percentile of the noncentral t-distribution with $n-1$ degrees of freedom and noncentrality parameter $-n^{\frac{1}{2}} K_{c}$. Resnikoff (1962) provides tables which facilitate the calculation of $t_{0}$. Alternatively, one may obtain an approximate value for $k_{2}$ by using the fact that $\bar{x}+k_{2} s$ has approximately a normal distribution (Jennett and Welsh, 1939). This yields

$$
k_{2}=\frac{C \cdot K_{c} \pm\left\{C^{2} K_{c}^{2}-\left(K_{1-\gamma}^{2} D-C^{2}\right)\left[\left(K_{1-\gamma}^{2} / n\right)-K_{c}\right]^{2}\right\}^{\frac{1}{2}}}{C^{2}-K_{1-\gamma}^{2} D}
$$

where $C=\sqrt{2} \Gamma(n / 2) /\left\{(n-1)^{\frac{1}{2}} \Gamma[(n-1) / 2]\right\}, D=1-C^{2}$ and the sign before the square root is positive if $\gamma>.5$ and negative otherwise.

In order to depict the OC surface given by (1) in two dimensions, the probability of acceptance may be plotted against $p_{1}$ for representative values of $p_{2}$, resulting in a separate curve for each value of $p_{2}$. The bivariate noncentral t-distribution is used to calculate the probability of acceptance when $0<\mathrm{p}_{2}<\mathrm{p}_{1}<1$. Details are given in the appendix. If either $\mathrm{p}_{2}=0$ or if $\mathrm{p}_{1}=\mathrm{p}_{2}$, then the noncentral t-distribution is used to calculate the probability of acceptance with $k=k_{1}$ and $p=p_{1}$ if $p_{2}=0$ and with $k=k_{2}$ and $p=p_{2}$ if $p_{1}=p_{2}$. Young and Minder (1972), using results of Owen (1965), provide an efficient algorithm to evaluate this integral. An APL program which 1mplements this algorithm may be obtained 
from the first author. Alternatively, Brown (1984) has shown that a normal approximation to $\bar{x}+k s$ provides an approximation to the above integral which is accurate to within .015 when $n \geq 5(k=1,2 ; p=.01, .05, .10, .20$, $.30, .40, .50)$. The approximation is the probability that

$$
z<\left(K_{p}-k C\right) /\left(1 / n+k^{2} D\right)^{\frac{1}{2}}
$$

where $\mathrm{Z}$ has a standard normal distribution.

\section{AN EXAMPLE}

Step 1: Suppose that $\alpha=.05, \beta=.10, \gamma=.10, a=.10, b=.30$ and $c=.05$. The resulting three points on the OC surface are $(.10,0, .95),(.30,0, .10)$ and $(.05, .05, .10)$.

Step 2: Using Hamaker's method, the values of $n$ and $k_{1}$ which satisfy the first two points approximately are $n=21$ and $k_{1}=0.867$.

Step 3: The value of $k_{2}$ which, in conjunction with $n=21$, satisfies the third condition given above is $k_{2}=2.196$. Therefore, in this example, the three-class procedure for sampling by variables is to take a random sample of 21 observations on the quality characteristic. Then accept the lot only if both $\bar{x}+0.867 \mathrm{~s} \leq \mathrm{U}_{1}$ and $\bar{x}+2.196 \mathrm{~s} \leq \mathrm{U}_{2}$ are satisfied and reject it otherwise. The operating characteristic surface for the above plan is illustrated in Figure 1. Note that for a particular value of $p_{1}$, this sampling procedure discriminates amongst lots with different values of $p_{2}$. When $p_{1}=0.10$, for example, the probability of acceptance is $95 \%$ for $p_{2}=0$ as compared to $24 \%$ for $\mathrm{P}_{2}=0.03$. A two-class plan could not discriminate between these two lot qualities. It is also eastly verified that the OC surface does indeed pass through the spectfled three points. 
4. MATCHING THREE-CLASS VARIABLES PLANS TO THREE-CLASS ATTRIBUTES PLANS Since three-class variables plans are proposed as alternatives to existing three-class attributes plans, it seems logical to develop variables plans for which the OC surfaces match the OC surfaces of specified attributes plans as closely as possible. Although it is generally not possible to devise a variables plan whose oc surface coincides exactly with that of a chosen attributes plan, it is possible, by the methods of the previous section, to choose a variables plan which matches at three suitably chosen points. For example, the $O C$ surface of the three-class attributes plan specified by $n=5, c_{1}=1$ and $c_{2}=0$ passes through the three points $(.076,0, .05),(.584,0, .10)$ and $(.369, .369, .10)$. However, if the use of a three-class variables procedure is justified, the plan specified by $\mathrm{n}=4, \mathrm{k}_{1}=0.558$ and $\mathrm{k}_{2}=1.380$ also provides the desired discrimination. The advantage of the variables procedure in this example is a $20 \%$ reduction in the sample size. The OC surfaces for these two procedures are illustrated in Figure 2. Although the two OC's are not identical, they both provide the desired discrimination and are approximately the same. Several examples of this matching procedure are summarized in Table 1 . In each case, the three points chosen for matching the variables plan to the attributes plan satisfy $\alpha=0.05, \beta=0.10$ and $\gamma=.10$. Note that in each case, the sample size required for the variables procedure is smaller than that required for the corresponding attributes procedure. The OC surfaces for the three-class attributes plan described by $n=50, c_{1}=1, c_{2}=0$ and for the three-class variables plan described by $n=24, k_{1}=1.903, k_{2}=2.211$ are illustrated in Figure 3. In this example of matching, the variables procedure requires less than half the sample size required for the attributes procedure to which it is designed to correspond. 


\section{THE $\left(\mathrm{p}_{1}^{*}, \mathrm{p}_{2}^{*}\right)-\mathrm{METHOD}$}

An alternative form of the decision rule in the proposed three-class variables plan would be, for a sample of size $n$ and with $p_{1}^{*}>p_{2}^{*}$, to accept the lot only if $\hat{p}_{1}<p_{1}^{*}$ and $\hat{p}_{2}<p_{2}^{*}$, and to reject it otherwise where

$$
\begin{aligned}
& \hat{\mathrm{p}}_{1}=\int_{0}^{\max \left[0, \mathrm{~W}_{\mathrm{U} 1}\right]} \mathrm{g}[\mathrm{t} ;(\mathrm{n} / 2)-1,(\mathrm{n} / 2)-1] \mathrm{dt} \\
& \hat{\mathrm{p}}_{2}=\int_{0}^{\max \left[0, \mathrm{~W}_{\mathrm{U} 2}\right]} \mathrm{g}[\mathrm{t} ;(\mathrm{n} / 2)-1,(\mathrm{n} / 2)-1] \mathrm{dt} \\
& \mathrm{W}_{\mathrm{U} 1}=(1 / 2)-\mathrm{n}^{\frac{1}{2}}\left(\mathrm{U}_{1}-\overline{\mathrm{x}}\right) /[2 \mathrm{~s}(\mathrm{n}-1)], \\
& \mathrm{W}_{\mathrm{U} 2}=(1 / 2)-\mathrm{n}^{\frac{1}{2}}\left(\mathrm{U}_{2}-\overline{\mathrm{x}}\right) /[2 \mathrm{~s}(\mathrm{n}-1)],
\end{aligned}
$$

and

$$
g(x: a, b)=\frac{\Gamma(a+b)}{\Gamma(a) \Gamma(b)} x^{a-1}(1-x)^{b-1} \quad \text { for } 0<x<1 \text {. }
$$

The quantities $\hat{\mathrm{p}}_{1}$ and $\hat{\mathrm{p}}_{2}$ are uniformly minimum variance unbiased estimators (UMVUEs) of the lot proportions above the marginal and defective specification limits, respectively, and $p_{1}^{*}$ and $p_{2}^{*}$ are two suitably chosen critical values. This method is equivalent to the $\left(k_{1}, k_{2}\right)$-method described in Section 2. The equivalence may be demonstrated as follows:

$$
\begin{aligned}
& \text { Let } \beta_{i *} \text { satisfy } \\
& \qquad \int_{0}^{\beta}{ }^{i *} g[t ;(n / 2)-1,(n / 2)-1] d t=p_{1}^{*} \quad \text { for } i=1 \text { and } 2 .
\end{aligned}
$$

Then the probability of acceptance of a lot with proportions $p_{1}$ above $U_{1}$ for $1=1,2$ will. be

$\hat{\mathrm{p}}\left(\hat{\mathrm{p}}_{1}<\mathrm{p}_{1}^{*}\right.$ and $\left.\hat{\mathrm{p}}_{2}<\mathrm{p}_{2}^{*} \mid \mathrm{p}_{1}, \mathrm{p}_{2}\right)$

$=P\left[.5-n^{\frac{1}{2}}\left(U_{1}-\bar{x}\right) / 2 s(n-1) \leq \beta_{1 *}\right.$ and $\left..5-n^{\frac{1}{2}}\left(U_{2}-\bar{x}\right) / 2 s(n-1) \leq \beta_{2 *} \mid p_{1}, p_{2}\right]$

$=P\left[T_{1 f}<2(n-1)\left[\beta_{1 *}-(1 / 2)\right]\right.$ and $\left.T_{2 f}<2(n-1)\left[B_{2 *}-(1 / 2)\right]\right]$, 
where $T_{\text {if }}$ is a noncentral $t$ random varlable with $f=n-1$ degrees of freedom and with noncentrality parameter $\delta_{i}=-n^{\frac{1}{2}} K_{p i}$ for $i=1$ and 2 . Hence (4) is equal to (1) if $-n^{\frac{1}{2}} k_{i}=2(n-1)\left[\beta_{i *}-(1 / 2)\right]$ for $i=1,2$. Thus we define $\beta_{1 *}=$ $(1 / 2)-n^{\frac{1}{2}} k_{1} /[2(n-1)]$ and $\beta_{2 *}=(1 / 2)-n^{\frac{1}{2}} k_{2} /[2(n-1)]$. Then the critical values $p_{1}^{*}$ and $p_{2}^{*}$ may be evaluated by substituting $\beta_{1 *}$ and $\beta_{2 *}$ into equation (3). This establishes the equivalence of the $\left(k_{1}, k_{2}\right)$-method and the $\left(\mathrm{p}_{1}^{*}, \mathrm{p}_{2}^{*}\right)$-method. The $\left(\mathrm{p}_{1}^{*}, \mathrm{p}_{2}^{*}\right)$-method has intuitive appeal over the $\left(k_{1}, k_{2}\right)$-method in that besides resulting in a decision about lot disposition, it also provides the inspector with UMVUEs of $p_{1}$ and $p_{2}$.

\section{CONCLUDING REMARKS}

A three-class procedure for acceptance sampling by variables has been introduced and illustrated with examples. The procedure, which requires that the distribution of the quality characteristic either be normal or transformable to a normal distribution, is suggested as an alternative to both the three-class attributes plan and the two-class variables plan. Its advantage over the three-class attributes procedure is a reduction in the sample size required for a plan with approximately the same oc surface while its advantage over the two-class variables procedure is a greater ability to discriminate when the nature of the quality characteristic conforms to the three-class definitions.

Two equivalent methods of stating the decision rule have been suggested for the three-class variables plan. The $\left(p_{1}^{*}, p^{*}{ }_{2}\right)$-method has intuitive appeal; besides resulting in acceptance or rejection of the lot, it also provides the inspector with uniformly minimum variance unbiased estimates of $p_{1}$ and $p_{2}$.

It has been shown that a special case of the bivariate noncentral t-distribution can be used to construct the oc surface for a three-class 
variables plan. The calculations required for the examples presented in this paper were carried out with a set of APL programmes which were written to aid the user in the choice of a plan (Brown, 1984). The availability of microcomputers and the potential avallability of interactive computer programmes for such procedures for sampling by variables thus eliminate one outdated advantage that the attributes plans have always held - their simplicity.

It has been demonstrated that in situations in which use of the three-class variables procedure is justified, the required sample size is usually smaller (and never larger) than that required for a corresponding three-class attributes procedure. In one of the examples presented, the sample size was reduced by more than $50 \%$.

\section{ACKNOWLEDGEMENTS}

This work was supported by a Natural Sciences and Engineering Research Council of Canada Operating Grant. The paper was written while the second author was a Visiting Fellow in the Biometrics Unit, Cornell University.

APPENDIX. EVALUATING THE OC SURFACE OF THE THREE-CLASS VARIABLES PLAN

Owen (1965) introduced the following function:

$$
Q_{f}(t, \delta ; v, w)=\frac{(2 \pi)^{\frac{1}{2}}}{\Gamma(f / 2) 2^{(f-2) / 2}} \int_{v}^{w} \Phi\left[\left(t y / f^{\frac{1}{2}}\right)-\delta\right] y^{f-1} \phi(y) d y
$$

where $\phi(\cdot)$ is the density of the standard normal distribution and $\phi(\cdot)$ is its cumulative distribution function. It can then be shown that for $t_{1}>t_{2}$ and $\delta_{1}>\delta_{2}$, which is the case of interest in this paper

$$
P\left(T_{1 f} \leq t_{1} \text { and } T_{2 f} \leq t_{2} \mid \delta_{1}, \delta_{2}\right)=Q_{f}\left(t_{1}, \delta_{1} ; 0, R\right)+Q_{f}\left(t_{2}, \delta_{2} ; R, \infty\right)
$$

where

$$
R=\left(\delta_{1}-\delta_{2}\right) /\left(A_{1}-A_{2}\right), \quad A_{1}=t_{1} / f^{\frac{1}{2}} \text { and } A_{2}=t_{2} / f^{\frac{1}{2}}
$$


Evaluation of (5) and (6) for particular values of $t, \delta, R$, and $f$ may be accomplished by repeated integration by parts. The resulting formulae are given by Owen (1965). An APL program is available from the first author to evaluate $(6)$.

\section{REFERENCES}

BRAY, D. F., LYON, D. A., and BURR, I. W. (1973), "Three Class Attributes Plans in Acceptance Sampling," Technometrics, 15, 575-585.

BROWN, P. A. (1984), A Three-Class Procedure for Acceptance Sampling by Variables, M.Sc. Thesis, Guelph, Ontario: University of Guelph. HAMAKER, H. C. (1979), "Acceptance Sampling for Percent Defective by Variables and by Attributes," Journal of Quality Technology, 11, 139-148.

ICMpF (1978), Microorganisms in Foods 2: Sampling for Microbiological Analysis, Principles and Specific Applications, International Commission on Microbiological Specification for Foods, edited by F. S. Thatcher and D. S. Clark, Toronto, Canada: University of Toronto Press.

JENNETT, W. J., and WELCH, B. L. (1939), "The Control of Proportion Defective as Judged by a Single Quality Characteristic Varying on a Continuous Scale," Journal of the Royal Statistical Soc., Ser. B 6 , 80-88.

KAO, J. H. K. (1971), "MIL-STD-414 Sampling Procedures and Tables for Inspection by Variables for Percent Defective," Journal of Quality Technology 3, 28-37.

KOYOMA, T., OHMAE, Y., SUGA, R., YOKOH, T., and YAMAMOTO, T. (1970), "MIL-STD-105D and the Japanese Modified Standard," Journal of Quality Technology 2, 99-108. 
OWEN, D. B. (1965), "A Special Case of the Bivariate Non-Central tDistribution," Blometrika, 52, 437-446.

RESNIKOFF, G. J. (1962), "Tables to Facilitate the Computation of Percentage Points of the Non-Central t-Distribution," Annals of Mathematical Stat1stics, 33, 580-586.

WALLIS, W. A. (1947), "Use of Vartables in Acceptance Inspection for Percent Defective," in Selected Techniques of Statistical Analysis for Scientific and Industrial Research and Production and Management Englneer1ng, New York: McGraw-Hill Book Company, Inc.

YOUNG, J. C., and MINDER, C. E. (1972), "Algorithm AS 76. An Integral Useful in Calculating Non-central $t$ and Blvarlate Normal Probabilities," Applied Statistics, 23, 455-457. 


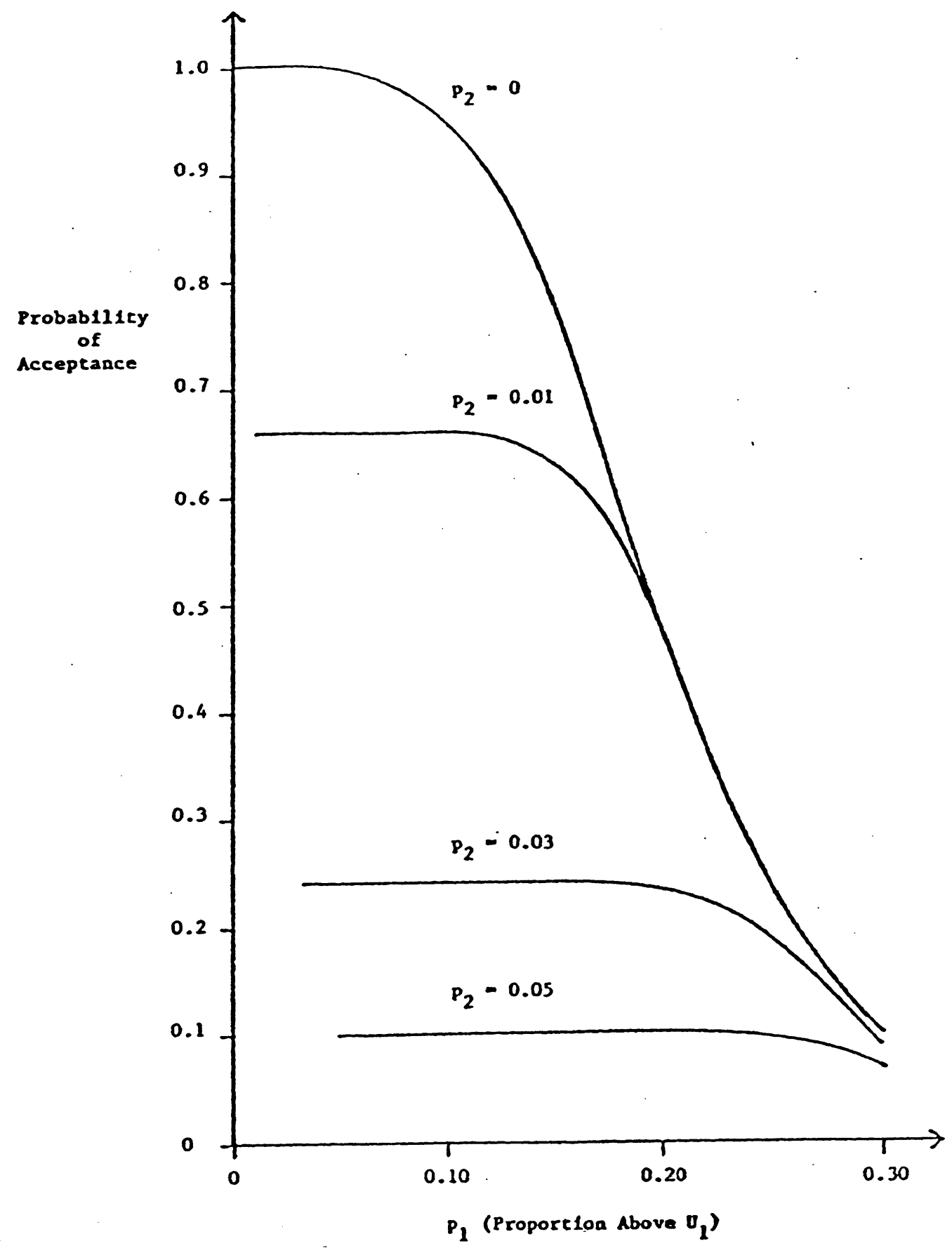

Figure 1. The oC surface for the three-class varlables plan described by $n=21, k_{1}=0.867$, and $k_{2}=2.196$. 


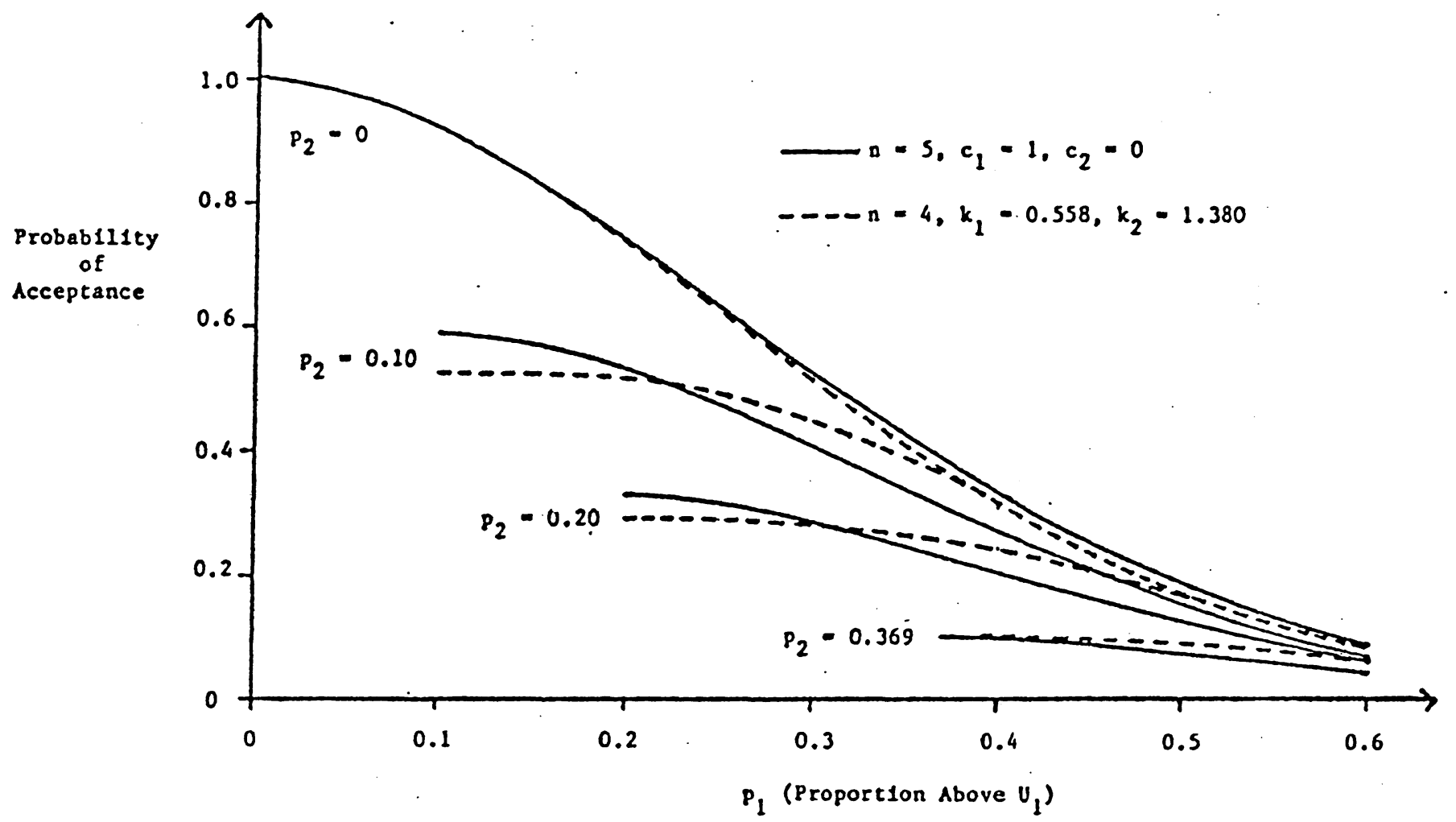

Figure 2. The oC surfaces for the three-class attributes plan described by $n=5, c_{1}=1$ and $c_{2}=0$ and the corresponding three-class variables plan described by $n=4, k_{1}=0.558$ and $k_{2}=1.380$. 
Table 1. Examples of Three-Class Variables Plans Chosen to Correspond to Three-Class Attributes Plans

\begin{tabular}{|c|c|c|}
\hline $\begin{array}{l}\text { Three-Class } \\
\text { Attributes } \\
\text { Plan }\end{array}$ & $\begin{array}{c}\text { Three Points } \\
\text { Matched } \\
\left(p_{1}, p_{2}, p_{a}\left(p_{1}, p_{2}\right)\right)\end{array}$ & $\begin{array}{l}\text { Corresponding } \\
\text { Three-Class } \\
\text { Variables Plan }\end{array}$ \\
\hline $\begin{aligned} \mathrm{n}=5, \mathrm{c}_{1} & =1 \\
\mathrm{c}_{2} & =0\end{aligned}$ & $\begin{array}{r}(0.076,0,0.95) \\
(0.584,0,0.10) \\
(0.369,0.369,0.10)\end{array}$ & $\begin{aligned} n=4, k_{1} & =0.558 \\
k_{2} & =1.380\end{aligned}$ \\
\hline 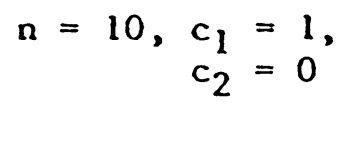 & $\begin{array}{r}(0.037,0,0.95) \\
(0.337,0,0.10) \\
(0.206,0.206,0.10)\end{array}$ & $\begin{aligned} \mathrm{n}=8, \mathrm{k}_{1} & =1.059 \\
\mathrm{k}_{2} & =1.571\end{aligned}$ \\
\hline $\begin{aligned} \mathrm{n}=10, & c_{1}=3 \\
c_{2} & =0\end{aligned}$ & $\begin{array}{r}(0.150,0,0.95) \\
(0.552,0,0.10) \\
(0.206,0.206,0.10)\end{array}$ & $\begin{aligned} n=7, k_{1} & =0.397 \\
k_{2} & =1.659\end{aligned}$ \\
\hline $\mathrm{n}=25, \begin{array}{l}\dot{c}_{1}=1 \\
c_{2}=0\end{array}$ & $\begin{array}{r}(0.014,0,0.95) \\
(0.147,0,0.10) \\
(0.088,0.088,0.10)\end{array}$ & $\begin{aligned} n=16, k_{1} & =1.580 \\
k_{2} & =1.937\end{aligned}$ \\
\hline $\mathrm{n}=50, \begin{array}{l}c_{1}=1, \\
c_{2}=0\end{array}$ & $\begin{array}{r}(0.007,0,0.95) \\
(0.076,0,0.10) \\
(0.045,0.045,0.10)\end{array}$ & $\begin{aligned} n=24, k_{1} & =1.903 \\
k_{2} & =2.211\end{aligned}$ \\
\hline $\begin{aligned} \mathrm{n}=50, & \mathrm{c}_{1}=3, \\
\mathrm{c}_{2} & =0\end{aligned}$ & $\begin{array}{r}(0.028,0,0.95) \\
(0.129,0,0.10) \\
(0.045,0.045,0.10)\end{array}$ & $\begin{aligned} \mathrm{n}=31, k_{1} & =1.486, \\
k_{2} & =2.134\end{aligned}$ \\
\hline
\end{tabular}




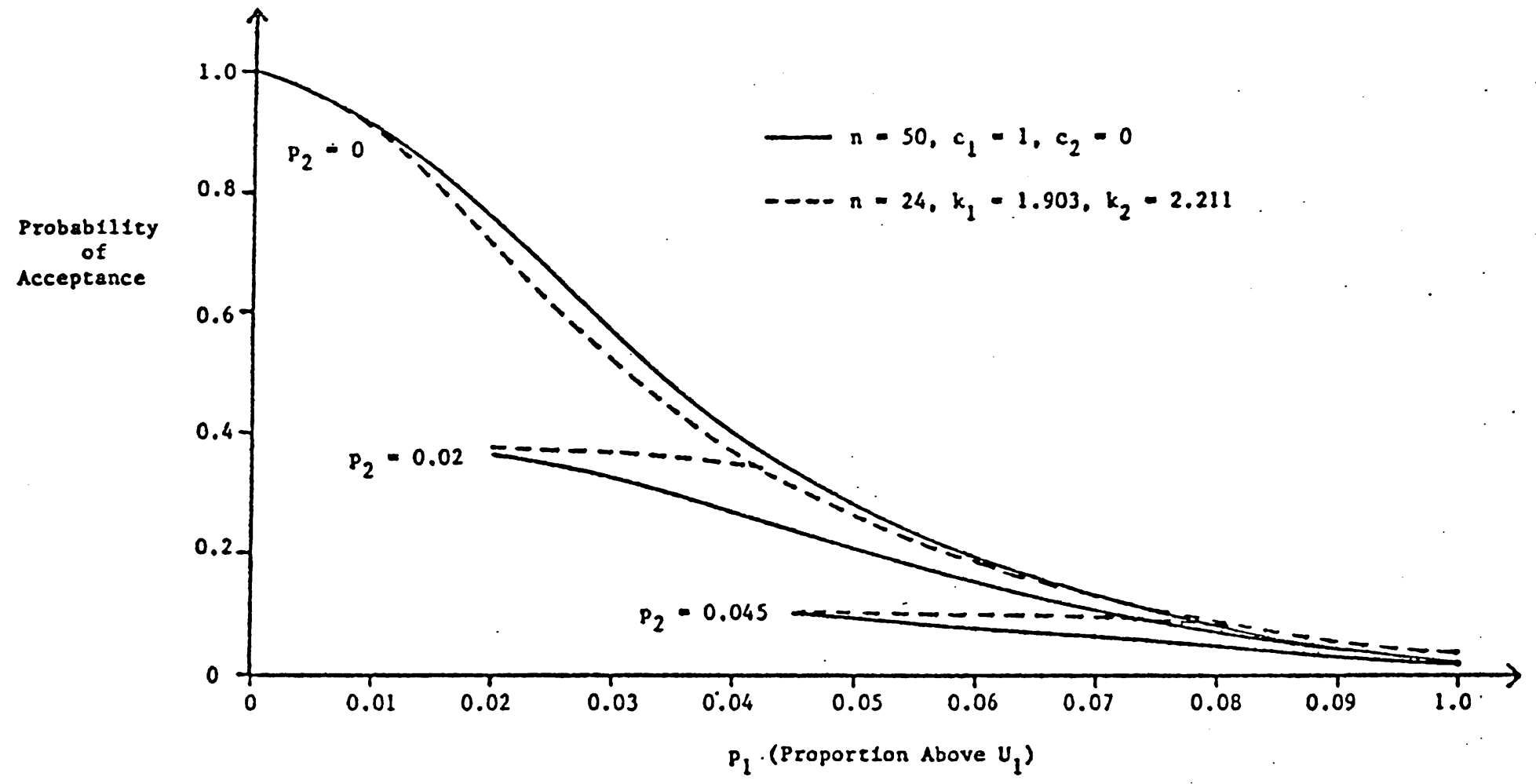

Figure 3. The oC surfaces for the three-class attributes plan described by $n=50, c_{1}=1$ and $c_{2}=0$ and the corresponding three-class variables plan described by $n=24, k_{1}=1.903$ and $k_{2}=2.211$ 